\title{
CONDICIONES METEOROLÓGICAS Y MATERIAL PARTICULADO PM1O Y PM2.5 EN LA CONSTRUCCIÓN DEL HOSPITAL HIPÓLITO UNÁNUE, TACNA 2019
}

METEOROLOGICAL CONDITIONS AND PARTICULATE MATERIAL PM10 AND PM2.5 IN THE CONSTRUCTION OF THE HOSPITAL HIPÓLITO

UNÁNUE, TACNA 2019

Diego Motocanche Ayala ${ }^{1}$ Richard Sabino Lazo Ramos²

Información del artículo:

Recibido: 05/05/2020

Aceptado: 15/06/2020

${ }^{1}$ Ingeniero Ambiental. E-mail: diegorena.ma@gmail.com

${ }^{2}$ Docente Facultad de Ingeniería, Universidad Privada de Tacna. E-mail: ozalsomar@gmail.com 


\section{Resumen}

El presente estudio se basó en la influencia de las condiciones meteorológicas en la dispersión de la concentración de los contaminantes atmosféricos PM10 y PM2.5 durante la construcción del Hospital Hipólito Unánue de Tacna en los meses de junio a setiembre de 2018. La metodología de muestreo y análisis estuvo basado en el protocolo de monitoreo de calidad de aire y gestión de datos de la DIGESA (2005). Las 2 estaciones de monitoreo EMHU01 y EMHU-02 registraron en el mes de junio para PM2.5 valores de 69.53 y 63,04 ug/m3 y en el mes de setiembre en la estación EMHU-01 el valor de 64,40 ug/m3 los cuales sobrepasaron el estándar de calidad ambiental para aire. Estos valores tuvieron relación directa con los altos valores de Temperatura de 18 y $19,6{ }^{\circ} \mathrm{C}$ y la velocidad de viento de $2,5 \mathrm{~m} / \mathrm{s}$ y una relación inversa con la Humedad relativa 76,3 y $75 \%$. Además se determinó que no existe relación significativa entre los datos obtenidos y ambas variables fueron corroboradas con la prueba estadística chi cuadrado de Pearson.

Palabras clave: Condiciones meteorológicas; Dispersión de Contaminantes; Material particulado.

\section{Abstract}

The study was based on the influence of meteorological conditions on the dispersion of PM10 and PM2.5 atmospheric pollutants during the construction of Hipólito Unánue Hospital in Tacna from June to September 2018. The sampling methodology and analysis was based on the DIGESA air quality monitoring and data management protocol (2005). The 2 monitoring stations EMHU-01 and EMHU-02 registered in the month of June for PM2.5 values of 69.53 and $63.04 \mathrm{ug} / \mathrm{m3}$ and in the month of September in the station EMHU-01 the value of $64.40 \mathrm{ug} / \mathrm{m} 3$ which exceeded the environmental quality standard for air. These values were directly related to the high temperature values of 18 and $19.6^{\circ} \mathrm{C}$ and the wind speed of $2.5 \mathrm{~m} / \mathrm{s}$ and an inverse relationship with the relative humidity of 76.3 and $75 \%$. In addition, the analysis of the variance determined that there is no significant difference between the data obtained and both variables were corroborated with the Pearson statistical test $(r)$ 0.705 , the correlation coefficient $p$-value $(s i g=0.016)$ and with a level of significance less than 0.05 .

Key words: Weather conditions; Pollutant Dispersion; Particulate matter. 


\section{Introducción}

Actualmente vivimos periodo de aumento, en la cual, el dinamismo de los habitantes ha causado consecuencias negativas, actividad que han impulsado al progreso industrial, agrario, económico, etc., siendo uno de las causas importantes en la evolución de la polución de la atmósfera del mundo en sus diferentes maneras. Las actividades urbanísticas también provocan inmensas cambios en el ambiente, el menoscabo del estado del aire en las ciudades, principalmente a causa del parque automotor, que es un problema mundial que se incrementa con el crecimiento de la población.

Entre los contaminantes del aire urbano, el material particulado el contaminante de la atmósfera más estudiado e importante a nivel mundial, siendo la agrupación de partículas sólidas y/o líquidas (excepto el agua pura) que están en la atmósfera, y poseen un amplio rango de propiedades morfológicas, físicas, químicas y termodinámicas. Estos polvos pueden ser dispersos al aire directamente cuando proceden de la combustión el cual es dispersado por el viento; una de las fuentes de generación de partículas en las ciudades es el tráfico, actividades que generan combustión dentro de su proceso, calles sin pavimento, construcciones, etc. Estas partículas causan efectos negativos sobre la salud a nivel de aparato respiratorio y el sistema cardiovascular. A su vez la presencia de este contaminante ocasiona variedad de impactos en el ambiente ya sea en la vegetación, materiales y al hombre, entre ellos, la disminución de la visión, con el incremento del esparcimiento y/o de la absorción de la radiación del sol, teniendo un efecto en la radiación de onda corta y con el incremento del cantidad de núcleos de condensación en el espacio. Existen pruebas de los perjuicios provocados por los depósitos de material particulado en infraestructura como edificaciones y obeliscos.

La Región Tacna presenta un elevado potencial de desarrollo, concentra cerca de 321 mil 351 habitantes, 40 mil vehículos y 17 mil 339 empresas con actividades de comercio, en la base de un área urbana que se extiende sobre una superficie de 16 mil 075 Kilómetros (INEI, 2016),

Actualmente la ciudad desarrolla su obra emblemática obra la construcción del nuevo Hospital Hipólito Unánue, la cual, generaría un impacto negativo directo sobre los parámetros del estado del aire por emisiones de material particulado que dañaría la salud de los obreros como a los pobladores que viven alrededor de la obra en construcción y así como de la posible intervención de las condiciones climáticas en la emisión del material particulado.

\section{Objetivos}

Evaluar si las condiciones meteorológicas influyen en la dispersión de las concentraciones de Material Particulado en la fase de construcción del Hospital Hipólito Unánue de Tacna.

\section{Metodología}

El tipo de investigación fue de tipo descriptiva según lo definen varios autores (Danke, 1986; Hernández, Fernández \& Baptista, 1997; Sampieri, 2010, Hernández, Fernández, Baptista, 2002, 2007), de tipo transversal (Hernández, Fernández y Baptista, 2000; Buendía, Collas \& Hernández, 1999).

Se realizó el monitoreo para determinar la relación que tienen las condiciones meteorológicas en la dispersión de concentraciones de PM 10 y PM 2,5 representativas en áreas de alta densidad de trabajo de la obra de construcción del nuevo Hospital Regional mediante la instalación de 2 puntos de monitoreo de aire y contrastando con el cumplimiento del Reglamento de Estándares Nacionales de Calidad Ambiental del Aire con fines tener una base de datos de emisiones y modelos de dispersión de contaminantes para el pronóstico de la calidad del aire. El monitoreo de aire y condiciones meteorológicas fue durante cuatro meses consecutivos, de Junio a Setiembre de 
2018, en la época de invierno.

Se midió temperatura $\left({ }^{\circ} \mathrm{C}\right)$, humedad $(\%)$, velocidad y dirección del viento $(\mathrm{m} / \mathrm{s}), \mathrm{PM}_{10}$ y $\mathrm{PM}_{2.5}(\mu \mathrm{g} / \mathrm{m} 3)$ por el método gravimétrico. La estrategia de monitoreo fue tomar los valores atmosféricos (material particulado y meteorología); según los protocolos de monitoreo de calidad de aire emitidos por la dirección general de salud (DIGESA); los análisis se realizaron en el laboratorio acreditado TYPSA sucursal del Perú, mediante métodos de ensayo específicos.

Se seleccionaron 2 estaciones de muestreo de aire, ubicados en diferentes puntos de la obra

A continuación se especifica el código y la descripción de los puntos de monitoreo así como la altura y la ubicación en coordenadas UTM (Universal Transverse Mercator) según lo observado en las tablas $1,2,3,4$.

Para el muestreo de Partículas en Suspensión se utilizó dos muestreadores de Alto Volumen PM10 Hi-Vol marca Thermo Scientific modelo Volumétrico (N/S P8087X) y marca Thermo Scientific modelo Volumétrico (N/S P9230X), los cuales captan partículas en suspensión con un diámetro aerodinámico menor a 10 micras, que succiona el aire del ambiente y pasa por un filtro. El análisis químico se realizó por gravimetría, los resultados se expresan en $\mu \mathrm{g} / \mathrm{m} 3$.

Para el muestreo de Partículas en Suspensión se utilizó un muestreador de Bajo Volumen PM2.5 Low Vol marca BGI modelo PQ 100 (N/S 0518), el cual, captan partículas en suspensión con un diámetro aerodinámico menor a 2.5 micras que succiona el aire del ambiente y lo pasa por un filtro de fibra de teflón. El análisis químico se realizó por gravimetría, los resultados se expresan en $\mu \mathrm{g} / \mathrm{m} 3$.

Para registrar diversas variables meteorológicas en un periodo de 24 horas, se utilizó dos estaciones meteorológicas marca Davis Instruments, modelo Vantage Pro 2 (N/S AZ170718039) y marca Davis Instruments modelo Vantage Pro 2 (N/S AZ170525074) las que midieron humedad, temperatura, velocidad y dirección del viento. Las cuales se recopilaron los datos meteorológicos (anexo 7).

\section{Tabla 1}

Ubicación de las estaciones de Aire mes de junio

\begin{tabular}{ccc}
\hline \multirow{2}{*}{ Datos } & \multicolumn{2}{c}{ Estaciones de monitoreo } \\
\cline { 2 - 3 } & EMHU-01 & EMHU-02 \\
\hline Fecha de muestreo & $09 / 06 / 2018$ \\
Hora de muestreo & Cerca del Auditorio & Zona de Carpintería \\
Localización & $367131 \mathrm{E}$ & $367159 \mathrm{E}$ \\
& $8007605 \mathrm{~N}$ & $8007708 \mathrm{~N}$ \\
Coordenadas UTM & Cuadrante 19 K & Cuadrante 19 K \\
Altura & $555 \mathrm{msnm}$ & $554 \mathrm{msnm}$ \\
\hline
\end{tabular}

Nota: Elaboración propia, 2018. 
Tabla 2

Ubicación de las estaciones de Aire Mes Julio

\begin{tabular}{ccc}
\hline \multirow{2}{*}{ Datos } & \multicolumn{2}{c}{ Estaciones de monitoreo } \\
\cline { 2 - 3 } & EMHU-02 & EMHU-01 \\
\hline Fecha de muestreo & $12 / 07 / 2018$ \\
Hora de muestreo & $09: 30$ & $10: 53$ \\
Localización & Frente a Oficinas & Centro de la Obra \\
& $367197 \mathrm{E}$ & $367089 \mathrm{E}$ \\
Coordenadas UTM & $8007655 \mathrm{~N}$ & $8007650 \mathrm{~N}$ \\
& Cuadrante $19 \mathrm{~K}$ & Cuadrante $19 \mathrm{~K}$ \\
Altura & $554 \mathrm{msnm}$ & $551 \mathrm{msnm}$ \\
\hline
\end{tabular}

Nota: Elaboración propia, 2018.

Tabla 3

Ubicación de las estaciones de Aire mes de Agosto

\begin{tabular}{ccc}
\hline \multirow{2}{*}{ Datos } & \multicolumn{2}{c}{ Estaciones de monitoreo } \\
\cline { 2 - 3 } & EMHU-02 & EMHU-01 \\
\hline Fecha de muestreo & $\mathbf{0 1 / 0 8 / 2 0 1 8}$ \\
Hora de muestreo & $09: 00$ & $10: 00$ \\
Localización & Frente a Oficinas & Piso Técnico \\
& $367203 \mathrm{E}$ & $367186 \mathrm{E}$ \\
Coordenadas UTM & $8007675 \mathrm{~N}$ & $8007659 \mathrm{~N}$ \\
& Cuadrante $19 \mathrm{~K}$ & Cuadrante $19 \mathrm{~K}$ \\
Altura & $553 \mathrm{msnm}$ & $550 \mathrm{msnm}$ \\
\hline
\end{tabular}

Nota: Elaboración propia, 2018.

Tabla 4

Ubicación de las estaciones de Aire mes de Setiembre 2018

\begin{tabular}{|c|c|c|}
\hline \multirow{2}{*}{ Datos } & \multicolumn{2}{|c|}{ Estaciones de monitoreo } \\
\hline & EMHU-01 & EMHU-02 \\
\hline Fecha de muestreo & \multicolumn{2}{|c|}{ 03/09/2018 } \\
\hline Hora de muestreo & 11:50 & 13:05 \\
\hline Localización & Frente a Oficinas & Cerca de la Puerta № 05 \\
\hline & $367202 \mathrm{E}$ & $366970 \mathrm{E}$ \\
\hline Coordenadas UTM & $8007675 \mathrm{~N}$ & $8007722 \mathrm{~N}$ \\
\hline & Cuadrante $19 \mathrm{~K}$ & Cuadrante $19 \mathrm{~K}$ \\
\hline Altura & 559 msnm & $545 \mathrm{msnm}$ \\
\hline
\end{tabular}

Nota: Elaboración propia, 2018. 


\section{Resultados}

Los resultados del monitoreo de la calidad del aire y concentración PM10 y PM2.5 se muestra en la tabla 5.

\section{Tabla 5}

Monitoreo del Material Particulado durante los meses de junio a setiembre de 2018

\begin{tabular}{|c|c|c|c|c|c|c|}
\hline $\begin{array}{c}\text { MATERIAL } \\
\text { PARTICULADO }\end{array}$ & ESTACIÓN & 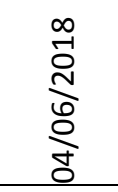 & 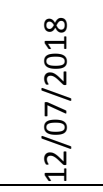 & $\begin{array}{l}\underset{\infty}{0} \\
\stackrel{0}{N} \\
\infty \\
\stackrel{0}{0} \\
-1\end{array}$ & 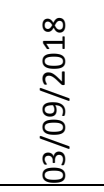 & $\begin{array}{c}\text { ECA } \\
\text { Aire } \\
\mathrm{ug} / \mathrm{m}^{3} \\
\end{array}$ \\
\hline \multirow{2}{*}{$\mathrm{PM}_{10}$} & EMHU-01 & 98,66 & 80,92 & 65,73 & 79,09 & \multirow[b]{2}{*}{$100(1)$} \\
\hline & EMHU-02 & 97,07 & 81,23 & 113,11 & 39,71 & \\
\hline \multirow[t]{2}{*}{$\mathrm{PM}_{2.5}$} & EMHU-01 & 69,53 & 35,18 & 41,58 & 64,40 & \multirow{2}{*}{$50(1)$} \\
\hline & EMHU-02 & 63,04 & 39,25 & 49,78 & 37,43 & \\
\hline
\end{tabular}

Nota. (1) Promedio diario. ECA Aire D.S. N003-2017-MINAM. Fuente; elaboración propia, 2018.

Los valores obtenidos para el parámetro material particulado menor a 10 micras $\left(\mathrm{PM}_{10}\right)$, en las estaciones monitoreadas EMHU-01 y EMHU-02, en el mes de setiembre EMHU - 01 supero los valores establecidos en los ECAs $\left(113,11 \mathrm{ug} / \mathrm{m}^{3}\right)$ para Aire de acuerdo al D.S. $\mathrm{N}^{\circ}$ 003-2017- MINAM. Los valores obtenidos para el parámetro material particulado menor a 2,5 micras $\left(\mathrm{PM}_{2.5}\right)$ en las estaciones EMHU- 01 y EMHU-02, en el mes de junio, superan el valor establecido $(69,53$ y 63,04 $\mathrm{ug} / \mathrm{m}^{3}$ ) respectivamente, y en el mes de setiembre en la estación EMHU-01 presenta el valor de 64,40 $\mathrm{ug} / \mathrm{m}^{3}$ ) excediendo también el ECA para Aire de acuerdo al D.S. Nº03- 2017-MINAM.

En los meses de Junio y Setiembre se presentó la temperatura promedio en las 2 estaciones de monitoreo EMHU-01 y EMHU-02 $\left(16,3^{\circ} \mathrm{C}\right)$, de igual manera en los valores más altos de humedad $(81 \%) ; y$ en los Valores altos de velocidad del viento $(2,7 \mathrm{~m} / \mathrm{s})$. Por otro lado, la predominancia de la dirección del viento para el mes de junio en la estación EMHU-01 fue de Noreste (NE) y en la estación EMHU-02 fue de predominancia Oeste.

Para contrastar si existe relación entre los Valores de material particulado y Condiciones meteorológicas, se comprobó mediante el chi-cuadrado de Pearson ( $p$-valor $=0,016$ ), que existe relación significativa, de formas similar los valores de PM10 con la temperatura ambiente, humedad relativa y velocidad del viento, se relacionan significativamente ( $p$-valor $=0,013)$, asi como los valores de $\mathrm{PM}_{2.5}$ con la temperatura ambiente, humedad relativa y velocidad del viento $(\mathrm{p}$-valor $=0$, 016), todos al $95 \%$ de confianza. 
Tabla 6

Condiciones meteorológicas durante los meses de junio a setiembre de 2018

\begin{tabular}{|c|c|c|c|c|c|c|c|c|c|c|c|}
\hline \multirow{2}{*}{$\begin{array}{c}\text { Fecha de } \\
\text { registro }\end{array}$} & \multirow{2}{*}{$\begin{array}{r}\text { Estación de } \\
\text { monitoreo }\end{array}$} & \multicolumn{3}{|c|}{$\begin{array}{c}\text { Temperatura } \\
\text { (oC) }\end{array}$} & \multicolumn{3}{|c|}{$\begin{array}{l}\text { Humedad } \\
\text { Relativa (\%) }\end{array}$} & \multicolumn{3}{|c|}{$\begin{array}{l}\text { Velocidad del viento } \\
(\mathrm{m} / \mathrm{s})\end{array}$} & \multirow{2}{*}{$\begin{array}{c}\text { Dirección } \\
\text { del } \\
\text { viento } \\
\text { promedio }\end{array}$} \\
\hline & & Prom & Máx & Mín & Prom & Máx & Mín & Prom & Máx & Mín & \\
\hline \multirow{2}{*}{$04 / 06 / 2018$} & EMHU-01 & 18 & 24 & 14 & 76,3 & 90 & 56 & 2,5 & 4,5 & 1,3 & NE \\
\hline & EMHU-02 & 19,6 & 27 & 14 & 75 & 89 & 65 & 2,5 & 3,6 & 1,3 & 0 \\
\hline \multirow{2}{*}{$12 / 07 / 2018$} & EMHU-01 & 15,6 & 24 & 12 & 74,9 & 90 & 48 & 2,7 & 4,5 & 0,9 & NO \\
\hline & EMHU-02 & 14,8 & 21 & 12 & 80 & 90 & 48 & 2 & 4 & 0,4 & NO \\
\hline \multirow{2}{*}{ 01/08/2018 } & EMHU-01 & 14,9 & 20 & 13 & 76,1 & 84 & 56 & 2,1 & 4 & 0,9 & NE \\
\hline & EMHU-02 & 13,5 & 19 & 12 & 81 & 89 & 60 & 2,4 & 4 & 1,3 & 0 \\
\hline \multirow{2}{*}{ 04/09/2018 } & EMHU-01 & 15,3 & 21 & 11 & 72,3 & 84 & 50 & 2,6 & 6,7 & 0 & 0 \\
\hline & EMHU-02 & 16,3 & 23 & 12 & 69,7 & 84 & 45 & 1,8 & 3,6 & 0 & 0 \\
\hline
\end{tabular}

Nota. EMHU; estación de monitoreo Hipolito Unanue. Fuente; elaboración propia, 2018.

\section{Discusiones}

Los valores obtenidos para el parámetro material particulado menor a 10 micras $\left(\mathrm{PM}_{10}\right)$, en las estaciones monitoreadas EMHU-01 y EMHU-02, estuvieron dentro del rango de los valores establecidos en el ECA para aire de acuerdo al D.S. $N^{\circ}$ 003-2017- MINAM. Comparando con los parámetros monitoreados por la Municipalidad Provincial de Tacna y SENAMHI (2009) se registró para Material Particulado $\mathrm{PM}_{10}$ en el Mercado Modelo $85,046 \mu \mathrm{g} / \mathrm{m}^{3}$., mientras que en un monitoreo realizado por el Gobierno Regional de Tacna (2014) fue 66,3 $\mathrm{gg} / \mathrm{m} 3$., destacando que dos puntos de monitoreo en el presente estudio no sobrepasaron los ECA., asi como en otros puntos monitoreados en la ciudad, tales como el monitoreo realizado por el Gobierno Regional de Tacna (2014), en los distritos de Tacna, Ciudad Nueva, Alto de la alianza y coronel Gregorio Albarracín Lanchipa de la Provincia de Tacna, se obtuvieron niveles de PM10 Centro de Salud Ciudad Nueva $\left(73,1 \mu \mathrm{g} / \mathrm{m}^{3}\right)$, Gran Hotel Tacna $\left(53 \mu \mathrm{g} / \mathrm{m}^{3}\right)$, Casino de la Policía Militar $\left(51,3 \mu \mathrm{g} / \mathrm{m}^{3}\right)$, y el ISTP Vigil $\left(47,8 \mu \mathrm{g} / \mathrm{m}^{3}\right)$ y en la ESPG UNJBG $(66,3 \mu \mathrm{g} / \mathrm{m3})$. Y parámetros monitoreados por la Municipalidad Provincial de Tacna y SENAMHI (2009) que registró para Material Particulado $\mathrm{PM}_{10}$ en el Botadero Municipal $60,986 \mu \mathrm{g} / \mathrm{m}^{3}$ y en el Parque Industrial 53,692 $\mu \mathrm{g} / \mathrm{m} 3$ ubicadas a sotavento de las fuentes principales de contaminación las cuales registraron mayores valores que las ubicadas a barlovento: ESPG Jorge Basadre 47,867 $\mu \mathrm{g} / \mathrm{m} 3$ y Zofra Tacna $42,113 \mu \mathrm{g} / \mathrm{m} 3$.

Por otro lado, según el monitoreo de calidad del aire realizado por el MINAM (2013-2014) determinaron en la ciudad de Tacna se presentó altos niveles de $\mathrm{PM}_{2,5}$ sobrepasando el ECA, mientras que los niveles de PM10 no excedieron los ECA. Según García en Lima Callao en el 2008 los contaminantes de material particulado PM10 y PM2.5 llegan a superar el valor límite indicado en el ECA nacional $(150 \mu \mathrm{g} / \mathrm{m} 3)$ y el PM2.5 al valor referencial $(65 \mu \mathrm{g} / \mathrm{m} 3)$.

En el estudio realizado por Herrera et al. en Costa Rica en el 2008 se presentaron concentraciones de PM10 significativamente superiores $(42-29 \mathrm{~g} / \mathrm{m} 3)$ a las registradas para zonas las zonas residenciales y comerciales de bajo flujo vehicular $(23-15 \mathrm{~g} / \mathrm{m3})$.

Según el trabajo realizado por Gómez et al. (2000) en la zona de Guayabal, Medellín, Colombia, 
las concentraciones diarias tuvieron como promedio $74 \mu \mathrm{g} / \mathrm{m} 3$ no superaron ninguna vez la norma diaria de $150 \mu \mathrm{g} / \mathrm{m} 3$ fijada por la Agencia de Protección Ambiental de los Estados Unidos (US EPA). En el trabajo: "Relación entre las Partículas Finas $\left(\mathrm{PM}_{2.5}\right)$ y Respirables (PM10) en la Ciudad de Medellín, en el 2007, los resultados del análisis muestran, en la mayoría de los casos hace pensar que probablemente se puede cumplir con la norma anual para PM10, pero no para PM2.5 (Londoño \& Vasco, 2008).

En el estudio realizado en Medellín y otros 9 municipios durante los años 2001 al 2007 las concentraciones de partículas respirables (PM10) se encontraron elevadas a niveles que exceden en 200-400\% los límites de precaución definidos por la Organización Mundial de la Salud y la tendencia es al empeoramiento a medida que crece la densidad vehicular (Bedoya \& Martínez, 2008).

Los valores obtenidos para el material particulado menor a 2,5 micras $\left(\mathrm{PM}_{2.5}\right)$ en las estaciones EMHU-01 y EMHU-02, superaron el valor establecido en los ECAs para Aire de acuerdo al D.S. N 0032017-MINAM en el mes de junio en las 2 estaciones EMHU-01 con $69.53 \mathrm{ug} / \mathrm{m} 3$ y en la EMHU-02 con $63,04 \mathrm{ug} / \mathrm{m3}$; $y$ en el mes de setiembre en la EMHU-01 con 64,40 ug/m3.

Comparando los valores obtenidos en el presente estudio con los datos obtenidos del parque automotor realizado por Miranda \& Merma (2017) ubicado en la ESPG UNJBG presentó un valor de 33,6 $\mu \mathrm{g} / \mathrm{m} 3$.

En el monitoreo de la calidad ambiental realizado por el Gobierno Regional de Tacna (2014), en la Escuela de Postgrado se registró $(25,8 \mu \mathrm{g} / \mathrm{m} 3)$. Además, en los distritos de Tacna, Ciudad Nueva, Alto de la Alianza y Cnel. Gregorio Albarracín Lanchipa de la Provincia de Tacna se obtuvieron los niveles de PM2,5 en el Centro de Salud del distrito de Ciudad Nueva $(36,1 \mu \mathrm{g} / \mathrm{m} 3)$, en el Gran Hotel Tacna $(32,4$ $\mu \mathrm{g} / \mathrm{m3})$ y en el Cuartel de la Policía Militar $(26,6 \mu \mathrm{g} / \mathrm{m3})$.

Comparando con el trabajo realizado por la Municipalidad Provincial de Tacna y SENAMHI (2009), se observó que los valores registrados en el Mercado Modelo de la ciudad de Tacna 81,255 $\mu \mathrm{g} / \mathrm{m} 3$, superan en $67 \%$ al Estándar correspondiente para 24 horas.

Durante el mes de Junio, la predominancia de la dirección del viento en la estación EMHU-01 es de Noreste (NE) y velocidad promedio de $2,5 \mathrm{~m} / \mathrm{s}$, siendo las 18:00 horas donde se registró la mayor velocidad de viento llegando al pico de $4,5 \mathrm{~m} / \mathrm{s}$. La predominancia de la dirección del viento en la estación EMHU-02 es de Oeste (W) y velocidad promedio de 2,5 m/s, siendo las 05:00 y 07:00 horas donde se registró la mayor velocidad de viento llegando al pico de 3,6 m/s.

Durante el mes de Julio, La predominancia de la dirección del viento en la estación EMHU-02 es de Noroeste (NW) y velocidad promedio de $2,0 \mathrm{~m} / \mathrm{s}$, siendo las 10:00 y 04:00 horas donde se registró la mayor velocidad de viento llegando al pico de $4,0 \mathrm{~m} / \mathrm{s}$.

La predominancia de la dirección del viento en la estación EMHU-01 es de Noroeste (NW) y velocidad promedio de $2,7 \mathrm{~m} / \mathrm{s}$, siendo las 13:00 horas donde se registró la mayor velocidad de viento llegando al pico de $4,5 \mathrm{~m} / \mathrm{s}$.

Durante el mes de Agosto, la predominancia de la dirección del viento en la estación EMHU-02 es de Oeste (W) y velocidad promedio de $2,4 \mathrm{~m} / \mathrm{s}$, siendo las $12: 00$ y 17:00 horas donde se registró la mayor velocidad de viento llegando al pico de $4,0 \mathrm{~m} / \mathrm{s}$.

La predominancia de la dirección del viento en la estación EMHU-01 es de Noreste (NE) y velocidad promedio de $2.1 \mathrm{~m} / \mathrm{s}$, siendo las 16:00 horas donde se registró la mayor velocidad de viento llegando al pico de $4,0 \mathrm{~m} / \mathrm{s}$.

Durante el mes de Setiembre, a predominancia de la dirección del viento en la estación EMHU01 es de Oeste (W) y velocidad promedio de 2,6 m/s, siendo las 17:00 horas donde se registró la mayor 
velocidad de viento llegando al pico de

$6,7 \mathrm{~m} / \mathrm{s}$. La predominancia de la dirección del viento en la estación EMHU-02 es de Oeste (W) y velocidad promedio de $1.8 \mathrm{~m} / \mathrm{s}$, siendo las $12: 00,14: 00$ y 15:00 horas donde se registró la mayor velocidad de viento llegando al pico de $3,6 \mathrm{~m} / \mathrm{s}$.

Respecto a las condiciones climatológicas en la dispersión del material particulado, existe una correlación entre las diferentes fracciones del material particulado, que corresponden al PST, PM10 y PM2.5 Es claro que las partículas suspendidas totales - PST, están constituidas por el total de partículas suspendidas en el aire, mientras que las partículas suspendidas con diámetro menor a diez micras PM10 corresponden a una fracción del PST y a su vez, las partículas suspendidas con diámetro menor a 2,5 micras corresponden a una fracción del PM10. Con base en lo anterior se puede afirmar que la concentración de PM10 nunca podrá ser mayor que la concentración de PST y a su vez la concentración de PM2,5 no podrá sobrepasar a las de PM10 y PST.

\section{Conclusiones}

En los 2 puntos de monitoreo de aire, ubicado en el Hospital Regional, en los meses de junio a setiembre de 2018 para PM10 registró valores de 65,73 a $98.66 \mu \mathrm{g} / \mathrm{m} 3$ valores inferiores a los contemplados por los ECA DS 003-2017-MINAM $(100 \mu \mathrm{g} / \mathrm{m3})$ a excepción del mes de agosto $(113,11$ $\mu \mathrm{g} / \mathrm{m3}$ ) que sobrepaso según el ECA, excediendo en todos sus registros el valor guía de la OMS (50 $\mu \mathrm{g} / \mathrm{m3})$. Mientras que para PM2.5 sobrepasaron el ECA en el mes de junio $(69,53 \mu \mathrm{g} / \mathrm{m} 3)$, EMHU $02(63,04 \mu \mathrm{g} / \mathrm{m} 3)$ y en setiembre $(64,40 \mu \mathrm{g} / \mathrm{m} 3)$.

Las dos estaciones climatológicas, ubicadas en el Hospital Regional, registraron en los meses de junio a setiembre valores altos de humedad relativa ( $81 \%)$ en el mes de agosto, velocidad de viento $(2,7$ $\mathrm{m} / \mathrm{s})$ en el mes de julio y niveles regulares de temperatura $\left(13,5\right.$ a $\left.19,6{ }^{\circ} \mathrm{C}\right)$ en los meses de julio a setiembre.

También existe correlación entre los valores de PM2.5 y PM10 con los registros altos de humedad relativa velocidad del viento y con la temperatura durante la construcción del Hospital Regional de Tacna, con un nivel del $95 \%$ de confianza.

\section{Referencias Bibliográficas}

Ambiente, D. G. (2013). Plan de Acción para la mejora de la calidad del aire en la zona de atención Prioritaria de la Cuenca Atmosférica de Tacna. Tacna.

Dankhe, O. L. (1986). Investigación y comunicación, en C. Fernández-Collado y G.L.Dankhe (Eds): “La comunicación humana: ciencia social’. México, D.F: McGrawHill deMéxico. Capítulo 13, pp. 385 454.

Diario el peruano. (2001). "Reglamento de Estándares Nacionales de Calidad Ambiental del Aire”, D.S. № 074-2001-PCM del 21 de Junio del 2001.

Diario el peruano. (2008). "Estándares Nacionales de la calidad Ambiental del Aire"- Decreto supremo № 003-2008-MINAM del 22 de Agosto del 2008.

DIGESA. (2005). Protocolo de monitoreo de la calidad del aire y gestión de los datos.

R.D. № 1404/2005/DIGESA/SA.

Hernández Sampieri, Roberto; Fernández Collado, Carlos; Baptista Lucio, Pilar. (1997). Metodología de la Investigación. Mc Graw Hill, México. Disponible en:

Ingeniería Investiga Vol. 2 (1) Enero - diciembre 2020 
http://es.scribd.com/doc/415928/Hernandez-Sampieri-R-cap-2-45.Consulta: 24/07/2010.

Hernández Sampieri, Roberto; Fernández Collado, Carlos; Baptista Lucio, Pilar. (2000). Metodología de la Investigación. Mc Graw Hill, México, pp. 1. Disponible en: http://catarina.udlap.mx/u_dl_a/tales/documentos/lad/jasso_b_m/capitulo3.pdf.Consul ta: 24/07/2010.

Hernández, C, C. Fernández, P. Baptista. (2002). Metodología de la investigación. México: McGraw-Hill. Hughes, G, D. Mckee y C. Singler.

Hernández R, Fernandez-Collado C, Baptista P. (2007) Metodología de la investigación, Edit Mc Graw Hill, México D.F.

Herrera, J., Rojas, J. F., Beita, V. H., Rodríguez, A., \& Argüello, D. S. (2015). “Concentración y composición química de partículas PM10 en el área metropolitana de Costa Rica en 2012". Revista de Caiencias Abientales Tropical Journal of Environmental, 39-53.

INEI (2016). Departamento de Tacna cuenta con una población de 346 mil habitantes. Obtenido de Departamento de Tacna cuenta con una población de 346 mil habitantes:

https://www.inei.gob.pe/prensa/noticias/departamento-de-tacna-cuenta-con-unapoblacion-de-346-mil-habitantes-9270/

Ministerio del Ambiente. (2013-2014). Informe Nacional de la Calidad del Aire. Lineamientos de política de calidad del Aire. Viceministerio de Gestión Ambiental.

SENAMHI. (2012). Servicio Nacional de Meteorología e Hidrología. SENAMHI. (2010). Servicio Nacional de Meteorología e Hidrología.

SENAMHI (2009).Reporte Anual de parámetros meteorológicos en la estación MAP- Jorge Basadre G. Tacna.

Zonificación Económica y Ecológica. (2010). Gobierno Regional de Tacna. Gerencia Regional de Recursos Naturales y Gestión del Medio Ambiente. Proyecto mejoramiento del Servicio de Monitoreo e Información Ambiental. Tacna nov. 